\title{
Social, health, education and economic effects of COVID-19 on adolescent girls in Kenya: Responses from the first round of adolescent data collection, August 2020-Kilifi
}

Population Council

Follow this and additional works at: https://knowledgecommons.popcouncil.org/departments_sbsr-pgy How does access to this work benefit you? Let us know!

\section{Recommended Citation}

"Social, health, education and economic effects of COVID-19 on adolescent girls in Kenya: Responses from the first round of adolescent data collection, August 2020-Kilifi," COVID-19 Research \& Evaluations brief. Nairobi: Population Council, 2020. 


\title{
Social, health, education and economic effects of COVID -19 on adolescent GIRLS in Kenya.
}

\author{
Responses from the first round of adolescent data collection on \\ August 2020. \\ Kilifi
}

Highlights

Adolescent knowledge of COVID-19 risk factors, symptoms and preventive behaviors in Kilifi was mixed. Most knew the main symptoms of fever (65\%), cough (77\%), and difficulty breathing (43\%). About half $(55 \%)$ knew you can be infected without showing symptoms. However, over half $(59 \%)$ incorrectly stated only people with symptoms are contagious, a quarter reported COVID-19 cannot spread in hot places (24\%), and $21 \%$ reported COVID was a punishment from god.

Almost all (92\%) of adolescents said they had been doing some learning or school work from home, mainly reading other books (49\%) or reading materials from school $(40 \%)$. The main reason for not doing any was that they were needed at home to help with chores $(37 \%)$ followed by unable to access the lessons provided (19\%).

Almost a quarter $(21 \%)$ of adolescents said they were participating in income generating activities since COVID-19; $32 \%$ of older adolescent boys and $20 \%$ of older adolescent girls. These are risk factors for dropping out or failing to return to schools when they reopen.

Food insecurity is a potential threat: almost half (45\%) were skipping meals a couple times per week and $58 \%$ said they received food through school before the closures, highest among older adolescent girls (64\%).

\section{Knowledge, Attitudes, and Perceived Risk of Infection}

Between August 15th-18th, 2020 a COVID-19 phone survey was completed with 1,603 10-19 year old adolescents in Kilifi, Kenya. The average age was 17 years, $75 \%$ were female and $17 \%$ have their own cell phone. Households were part of the Evaluation of the Nia Project cohort. Kilifi county is along the coast of Kenya, north of Mombasa. As the end of July 2020, there were 101 confirmed cases of COVID-19 in Kilifi.

- Knowledge: Overall, respondents in Kilifi correctly identified the elderly as at high risk if infected (55\%) but less so those with weakened immune systems (12\%), and almost half incorrectly stated children are at highest risk (43\%). Knowledge of fever $(65 \%)$, cough $(77 \%)$ and difficulty breathing $(43 \%)$ as symptoms was high. Over half $(55 \%)$ knew asymptomatic transmission was possible. Almost all (87\%) knew that people of all ages can become infected. A proportion believed COVID-19 cannot spread in hot places (24\%) and that it is a punishment from god $(21 \%)$; these myths were more commonly reported by younger adolescents.

- Prevention: Most adolescents (89\%) correctly identified handwashing as a method to prevent the spread of COVID-19, $84 \%$ identified wearing masks and $62 \%$ said standing 1 to 2 meters apart.

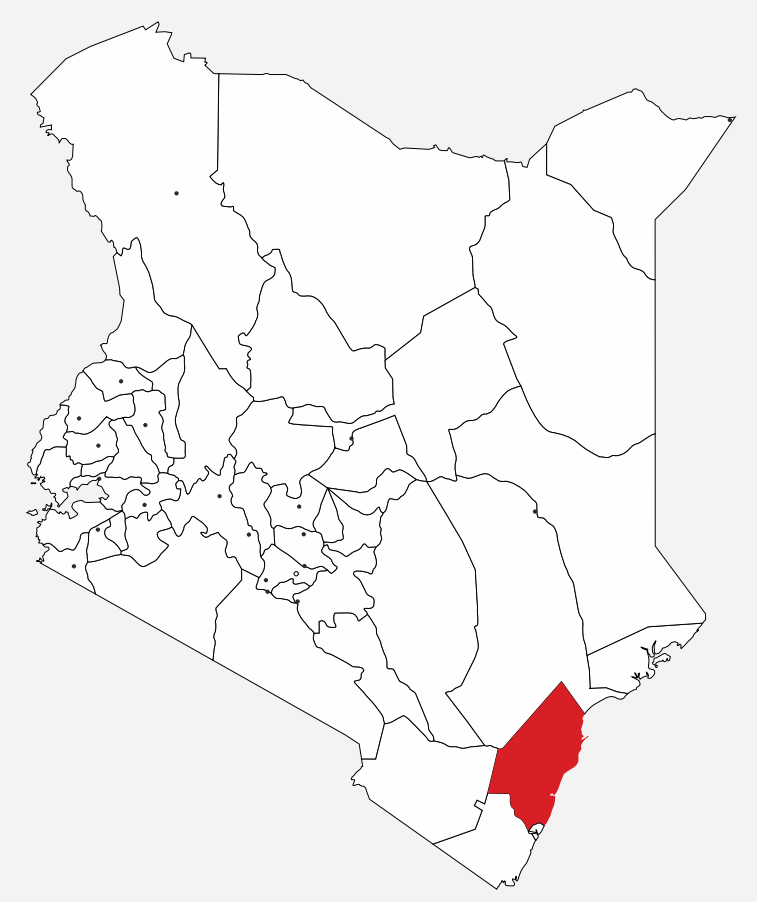


Information source: Participants reported getting information from Government radio advertisements (82\%), radio programs (74\%) and their friends (86\%). Only one third received information from government SMS.

- Perceived risk: Almost half of adolescents (42\%) believed their risk of contracting COVID-19 was low. Those with low perceived risk say it is because they wash their hands $(35 \%)$, wear a mask (35\%), practice physical distancing $(30 \%)$, and have been staying at home (28\%). Only $1 \%$ knew someone who had tested positive for COVID-19.

\section{COVID-19 Prevention Methods}

Two-thirds of adolescents went out of their home at least once in the last week. Of those that went out, 92\% wore a mask, $91 \%$ washed their hands upon returning home, and 76\% kept a distance of 1-2 meters. However, of those that went out, over half said they interacted with five or more people - this was higher for males than females.

- $20 \%$ said they rode public transportation when they went out, with little variation by age or gender.

\section{Impact of the COVID-19 Mitigation Response}

The COVID-19 pandemic and associated physical distancing and lockdown measures have had a large economic, health, educational, and social impact on respondents. Schools have been closed since March 2020, however many adolescents were able to continue their school-work. Those that were unable to do schoolwork cited the main reason is they were needed for household chores. Some were already engaged in income generating activities. These shifts away from education may make it less likely that adolescents return to school when they reopen.

\section{Education and Income Generating Activities.}

\footnotetext{
- Almost all (91\%) adolescents were in school before COVID-19 related closures, this was highest for younger adolescents, and lowest for older adolescent girls (89\%).

- When asked if they would return after school reopened, $94 \%$ said absolutely. The main reason for not returning was not being able to afford school feels $(83 \%)$.

Almost all (92\%) of adolescents said they had been doing some learning or school work from home, mainly reading other books $(49 \%)$ or reading materials from school $(40 \%)$. The main reason for not doing any was that they were needed to help with chores (37\%), they cant access the lessons provided (19\%), or the school hasn't provided any lessons (17\%).

- Very few adolescents were accessing school work via mobile phones (8\%) or the television/radio (8\%).

Over half of participants were spending more time on household chores such as cooking, cleaning, collecting water or firewood, or washing clothes. Girls spent more time on chores than boys, highest for older adolescent girls who report spending about 3.6 hours per day.

- Some were engaging in income generating activities since COVID-19 started, mostly older adolescents and more so males (32\% of older adolescent males, followed by $20 \%$ of older adolescent females). Most were not working or looking for work $(71 \%)$.
} 


\section{Decision Making}

- Most adolescents reported less decision-making power over what they do with money they earn (63\%), amount of time spent with friends (77\%), and the amount of time spent idling in the neighborhood 78\%) as compared to before COVID-19.

- Many participants have been skipping meals or eating less since the COVID-19 pandemic began. In the past 7 days, $45 \%$ said they are skipping meals a couple times per week, and $76 \%$ say this is more often than before COVID-19.

\section{Food Security}

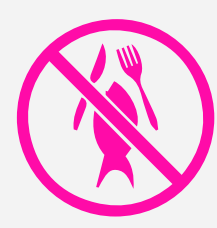

Many participants have been skipping meals or eating less since the COVID-19 pandemic began. In the past 7 days, $45 \%$ said they are skipping meals a couple times per week, and $76 \%$ say this is more often than before COVID- 19 .

- Before schools closed, $58 \%$ said they were receiving at least one meal at school. This was higher for older adolescents, and highest for older adolescent girls (64\%).

\section{Health and Safety}

- About a third of participants reported feeling down, depressed or hopeless, or feeling little interest or pleasure in things they normally do (34\%) at least once in the last two weeks; over half said they did not feel this way on any days. This was higher for older adolescents, highest for adolescent males.

- Of girls who have started menstruating, disposable sanitary pads are the most commonly used product, and over half $(57 \%)$ say they are having challenges getting their preferred product, mainly because they or their parents cannot currently afford it.

- About $9 \%$ of participants reported having been humiliated, insulted or threatened in the past one month, higher for males than females. About $4 \%$ overall reported having been hit, slapped, kicked or beaten in the last one month, highest for younger adolescent males (11\%).

- $99 \%$ of older adolescent girls are not currently pregnant and do not wish to get pregnant, $1 \%$ were pregnant or probably pregnant. Male condoms were the most commonly reported contraceptive method used $(3 \%)$. Only $18 \%$ were currently doing something to avoid or delay pregnant. The main reason for not using a method was that the participant is not currently sexually active, $9 \%$ say because they are abstinent. 


\section{Conclusions and Recommendations}

COVID-19 public education campaigns in Kisumu should continue with a focus on:

- Clarifying that one can both have COVID-19 and not show symptoms, as well as infect others even if asymptomatic

Addressing some of the common myths surrounding COVID-19.

Address the health, economic, and social impacts of lockdowns:

- Expand the options for students to access educational materials during the period of school closures so that they can maintain their skills and interest in learning. When schools open, pay close attention to older adolescents that have start working during COVID-19 to ensure that they re-enroll in school and complete secondary school.

- Adolescents are missing meals, especially since they are also not receiving meals from school during closures. Food distributions are necessary, targeting those were receiving more meals at school.

- Although most are not reporting that they feel depressed, many adolescents do feel anxious about COVID-19 and are seeing friends less than before. Ensuring they have accurate information and support is critical to improve mental health and prevent social isolation. 